\title{
Magnetization Controlled Superconductivity in a Film with Magnetic Dots.
}

\author{
Igor F.Lyuksyutov $^{a, *}$ and Valery Pokrovsky ${ }^{a, b}$ \\ (a) Department of Physics, Texas AEM University, College Station, TX 77843-4242
}

(b) Landau Institute for Theoretical Physics, Moscow, Russia

(January 13, 2014)

\begin{abstract}
We consider a superconducting film with Magnetic Dots Array (MDA) placed on it. Magnetic moments of these dots are supposed to be normal to the film and strong enough to create vortices in the superconducting film. Magnetic interaction between dots is negligible. Below the superconducting transition temperature of the film $T_{2 D}$ in zero magnetic field the MDA with randomly magnetized dots produces resistive state of the film. Paradoxically, in a finite magnetic field the film with MDA is superconductive.
\end{abstract}

74.60.Ge, 74.76.-w, 74.25.Ha, 74.25.Dw

Recently substantial progress has been achieved in the preparation of magnetic structures connected with superconducting films (see [1], [2], 3]). In experiments [2] a complex of a thin $(100 \mathrm{~nm})$ superconducting film and a single magnetic particle with typical dimension $10-100 \mathrm{~nm}$ was prepared by depositing micro-bridge-DCSQUID onto the particle [2]. This type of systems was used to study Macroscopic Quantum Tunnelling (MQT) [1] of the magnetic moment. In other type of experiments ( [3]) a superconducting film was placed on the top of a periodic array of magnetic dots with typical size and distance between dots $100 \mathrm{~nm}$. The magnetoresistance of the superconducting film oscillated as function of magnetic flux $\Phi$ through the unit cell of MDA [3] with the period equal to the magnetic flux quantum $\Phi_{0}$. In earlier experiments of this type [1], [5], [6] the square lattice of $200 \mathrm{~nm}$ thick magnetic particles was mounted upon $20 \mathrm{~nm}$ thin superconducting films. The magnetoresistance and transiton temperature of the superconducting film displayed periodic variation with magnetic field with the period corresponding to one flux quantum per unit cell.

Magnetic moment of magnetic dots can be oriented either parallel or normal to the surface of the substrate (superconducting film) in controllable way [7], 8]. In the cited experiments ( [1 3]) magnetic moment of dots was parallel to the film. The magnetic flux from nanomagnets in the direction normal to the superconducting film is readily available and it can be varied in a broad range from a few percent up to many flux quanta [9], [8] per a dot. Depending on the orientation of the dot magnetic moment and its strength, a single dot can create vortices or pairs of vortices, or it can pin the vortices in the superconducting film. With the help of nanomagnets one can create strong magnetic fields of various configurations on the surface with high precision .

The vortex-dot pairs interact through the magnetic field in vacuum and in superconducting film. The interaction between vortices in the superconducting film grows logarithmically with the distance, whereas the dipole- dipole interaction between magnetic moments in vacuum decreases with the distance. Thus, the magnetic dots bound with vortices interact at large distances mainly via vortices if the distance is still smaller than effective screening length $\lambda_{\text {eff }}$. According to Abrikosov ( [10], [11]), $\lambda_{\text {eff }}=\lambda^{2} / d$, where $\lambda$ is the London penetration depth and $d$ is the film thickness (see discussion below).

The phase state of the superconducting film with the magnetic dot lattice depends on the orientation of the dots magnetic moment with respect to the film and on the magnetic order in the array. Vortices coupled with magnetic dots create a "frozen" potential for individual vortices. If the dot magnetic moments are randomly oriented, this "frozen" potential fluctuates strongly. In deep fluctuations uncoupled vortices can appear spontaneously. As a result, the superconducting film may transit into the resistive state. However, if magnetic field aligns magnetic moments of the dots, deep random fluctuations of the vortex potential are suppressed. In a weak enough average magnetic field all vortices will be pinned by the dots. Paradoxically, the resistive state exists in zero field, while non-zero magnetic field restores superconductivity. Below we consider this phenomenon in more details.

The amplitude $J$ of the superconducting current density per unit area at distance $r \ll \lambda_{\text {eff }}$ from the vortex center is given by equation (see e.g. [11])

$$
J(\rho)=\frac{\phi_{0} c}{8 \pi^{2} \lambda_{e f f} r},
$$

where $\phi_{0}$ is the magnetic flux quantum and $r$ is the distance from the center. We assume for simplicity that the dot creates homogeneous magnetic field $H$ normal to the surface of superconducting film and localized in a circle of radius $R \ll \lambda_{e f f}$. The coupling energy $E_{d}$ between a vortex and the dot is:

$$
E_{d}=\int_{0}^{R} d r \pi r^{2} \frac{1}{c} J(r) H=\frac{H \phi_{0} R^{2}}{16 \pi \lambda_{e f f}}=\frac{\epsilon_{0} \phi_{d}}{\phi_{0}}
$$


where $\phi_{d}=\pi R^{2} H$ is the magnetic flux generated by the magnetic dot and $\epsilon_{0}=\phi_{0}^{2} / 16 \pi^{2} \lambda_{\text {eff }}$. The single vortex energy without magnetic field is (see e.g. [11]):

$$
E_{v}=\epsilon_{0} \ln \frac{l}{\xi}
$$

where $l$ is the characteristic cut-off length. The latter is equal to $\lambda_{\text {eff }}$ for a single vortex in the film and it is equal to $l=\min \left(a, \lambda_{e f f}\right)$ for a vortex lattice with the lattice constant $a$.

It follows from Eqs.(2):3) that the threshold value for magnetic flux per magnetic dot necessary to create a vortex is equal to

$$
\phi_{d c}=\phi_{0} \ln \frac{l}{\xi}
$$

In a typical experimental situation the logarithmic factor is not too large. The magnetic film must be separated from the superconducting film by an insulating layer (e.g. thin oxide film), to avoid the suppression of superconductivity by the proximity effect.

Two vortices with vorticities $n_{i}$ and $n_{j}\left(n_{i}, n_{j}= \pm 1\right)$ separated by the distance $r_{i j} \ll \lambda_{\text {eff }}$ interact logarithmically (see e.g. [16])

$$
V\left(r_{i j}\right)=-\frac{n_{i} n_{j} \phi_{0}^{2}}{16 \pi^{2} \lambda_{e f f}} \ln \frac{r_{i j}}{\xi}
$$

Eq.(5) demonstrates explicitly that, as we discussed earlier, the interaction between $\mathrm{MD}$ with magnetic moments normal to the film via attached vortices can be stronger than the direct magnetic dipolar interaction at large distances. Magnetic moment $\mathcal{M}$ of the magnetic dot with typical size $R$ is of the order of $\mathcal{M} \approx H R^{3}$, where $H$ is the magnetic field created by the dot. The dipolar magnetic interaction between two dots at distance $r$ can be estimated as $E_{\text {dip }}(r)=\phi_{0}^{2} R^{2} / r^{3}$, where we have used $H R^{2} \approx \phi_{0}$. Comparing this energy with Eq.(5) we find that the dipolar interaction between MD is negligible when $a \gg R\left(\lambda_{e f f} / R\right)^{1 / 3}$. The interaction between dots mediated by vortices changes substantially the magnetization curve when the film is cooled below the superconducting transition temperature. This statement can be verified experimentally.

Due to large potential barriers for spin reversal the magnetic moments in the MDA can be oriented randomly. Let thethe moments be large enough to create vortices at $T=0$. The vortices coupled to randomly oriented magnetic moments serve as sources of the frozen random potential $V(\mathbf{r})$ for a single vortex:

$$
V(\mathbf{r})=-\sum_{\mathbf{r}_{j}} n_{j} \epsilon_{0} K_{0}\left(\frac{\left|\mathbf{r}-\mathbf{r}_{j}\right|}{\lambda_{e f f}}\right)
$$

where $n_{j}$ is the vorticity, i.e. the random variable taking values \pm 1 , and $K_{0}(x)$ is the zero-order MacDonald function (see e.g. [12]). For the square MD lattice with the lattice constant $a$ the mean square fluctuation of this potential reads:

$$
\begin{aligned}
\left\langle V^{2}(\mathbf{r})\right\rangle & =\epsilon_{0}^{2} \sum_{\mathbf{r}_{j}, \mathbf{r}_{i}}\left\langle n_{i} n_{j}\right\rangle K_{0}\left(\frac{\left|\mathbf{r}-\mathbf{r}_{i}\right|}{\lambda_{e f f}}\right) K_{0}\left(\frac{\left|\mathbf{r}-\mathbf{r}_{i}\right|}{\lambda_{e f f}}\right) \\
& =\epsilon_{0}^{2} \sum_{\mathbf{r}_{j}} K_{0}^{2}\left(\frac{\left|\mathbf{r}_{j}\right|}{\lambda_{e f f}}\right)=\epsilon_{0}^{2} \frac{\lambda_{e f f}^{2}}{a^{2}} \int_{\mathbf{r}} K_{0}^{2}(r) \\
& +\epsilon_{0}^{2} \frac{\lambda_{e f f}^{2}}{a^{2}} \sum_{\mathbf{m} \neq \mathbf{0}} \int_{\mathbf{r}} K_{0}^{2}(r) \exp \left(2 \pi i \frac{\lambda_{\text {eff }}}{a} \mathbf{r} \cdot \mathbf{m}\right)
\end{aligned}
$$

We assumed uncorrelated vorticities $n_{i}$, i.e. $\left\langle n_{i} n_{j}\right\rangle=\delta_{i, j}$ and introduced the vector $\mathbf{m}$ with integer coordinates $m_{x}, m_{y}$. Eqn.7] allows to find a typical variation (depth of potential valleys) in the frozen potential:

$$
\Delta V\left(\lambda_{e f f}, a\right) \equiv \sqrt{\left\langle V^{2}(\mathbf{r})\right\rangle}=\sqrt{\pi / 2} \epsilon_{0} \lambda_{e f f} / a
$$

Fluctuations with such energy have maximal allowed linear size $r=\lambda_{e f f}$. The magnitude $\Delta V\left(\lambda_{e f f}, a\right)$ of a typical potential fluctuation is increased linearly with $\lambda_{\text {eff }}$. The energy of a single vortex grows with $\lambda_{\text {eff }}$ only logarithmically 15 18:

$$
U_{s}\left(\lambda_{e f f}\right)=\epsilon_{0} \ln \left(\lambda_{e f f} / \xi\right)+\epsilon_{\text {core }}
$$

where $\epsilon_{\text {core }} \sim \epsilon_{0}$ is the energy of the vortex core. Comparing the vortex energy Eq. 9 with the typical fluctuation Eq. 8 of the random potential, we see that the creation of vortex is favorable at $a<\lambda_{\text {eff }}$. It means that, for sufficiently dense MDA or sufficiently high temperature, deep valleys in the random potential will be filled by unbound vortices.

When $a>\lambda_{e f f}$, fluctuations of the random potential can not unbound vortex pairs. We discuss now the transition line $a\left(\lambda_{e f f}\right)$ between the phase with bound vortices (superconducting phase) and the vortex plasma (resistive phase). Let us introduce the MD density $n_{d}=a^{-2}$ and its critical value $n_{d}^{c}$ at which the transition to the plasma state occurs at a given $\lambda_{e f f}$. In the plasma phase the vortex density outside of MD is defined by the random potential of bound to MD vortices and by the interaction between free vortices. In the limit of small vortex density $n_{v} \ll \lambda_{e f f}^{-2}$ the average energy gain per vortex is the difference of the energy of single vortex creation Eqn 9 and the energy gain due to the random potential Eq. 8. The repulsion between vortices of the same sign filling a typical random potential valley increases the energy per vortex by the value proportional to the $\epsilon_{0} \lambda_{e f f}^{2} n_{v}$ (neglecting the logarithmic factor). Thus, the mean field equation for energy density has the form:

$$
E_{v}=n_{v}\left(\epsilon_{0} \ln \left(\frac{\lambda_{e f f}}{\xi}\right)+\epsilon_{\text {core }}-\sqrt{\frac{\pi}{2}} \epsilon_{0} \lambda_{e f f} \sqrt{n_{d}}\right)+\frac{B \epsilon_{0}}{2} n_{v}^{2} \lambda_{e f f}^{2}
$$


where $B$ is a numerical factor of the order of one. Assuming $\epsilon_{0} \gg \epsilon_{\text {core }}$, the equilibrium vortex density following from Eqn. 10 is:

$$
n_{v} \propto \lambda_{e f f}^{-1}\left(\sqrt{n_{d}}-\sqrt{n_{d}^{c}}\right)
$$

where $n_{d}^{c}\left(\lambda_{e f f}\right)$ defines the transition line:

$$
n_{d}=n_{d}^{c} \equiv \frac{2}{\pi \lambda_{e f f}^{2}} \ln ^{2} \frac{\lambda_{e f f}}{\xi} .
$$

In the experimental setup the effective penetration depth varies with temperature. Thus, the theory predicts the transition to resistive state in zero field at some temperature $T_{r}<T_{c}$ if the MD density $n_{d}$ is smaller than the value $\lambda_{\text {eff }}^{-2}(0) \ln ^{2} \frac{\lambda_{\text {eff }}(0)}{\xi}$ taken at zero temperature. In a real experiment the creation of vortex plasma can be very slow at $T \ll T_{2 D}$ due to potential barriers. These barriers are of the order of $\epsilon_{0}$ i.e. of $T_{2 D}$. We expect that in zero external magnetic field, far away from the transition line Eqn.12, the densities of vortices of both signs in the vortex plasma are the same $n_{v} \approx a^{-1} \lambda_{\text {eff }}^{-1}$.

Qualitatively one can realize the influence of random distribution of vorticities as an effective random field of the strength $\mathcal{F}=\sqrt{\pi / 2} \epsilon_{0} / a$ which acts on vortices. The problem of diffusion of a classical particle (vortex in our case) in a random field type potential have been studied in 20,21. Dimensionality $D=2$ is marginal for many types of random field type disorder. In our case the screening effectively stops the growth of the random potential at distances of the order of $\min \left(a, \lambda_{e f f}\right)$. We do not expect any measurable effects in the transport properties due to low dimensionality.

Resistivity in the vortex plasma state is defined by equation (see e.g. [18, 19]):

$$
\rho=2 \rho_{n} n_{v} \xi^{2} \approx \rho_{n} \frac{\xi^{2}}{a \lambda_{e f f}}
$$

where $\rho_{n}$ is the resistivity in the normal state.

In the absence of MDA in zero field the superconducting film undergoes the Berezinskii-Kosterlitz-Thouless transition [13,14] with the transition temperature $T_{2 D}=$ $\epsilon_{0} / 2$. $T_{2 D}$ defines characteristic temperature range in this problem. The Curie temperature $T_{C}$ for magnetic dots can be easily choosen in such a way that $T_{2 D} \ll T_{C}$. When MDA system is cooled below the Curie point in zero field, the magnetic moments of the dots are oriented randomly. In turn, the vortices in a superconducting film are also oriented randomly. We have shown that this random field can fluctuate in space so strongly that the process of spontaneous vortex-antivortex pair formation is allowed, leading to resistive state of the superconducting film in zero field at zero temperature. Applying an external magnetic field, one can order the magnetic moments of the dots. In the magnetized phase of the MDA all vortices bound with dots are of the same sign. The periodic array of vortices which are bound by MD or created by external magnetic field produce strong pinning of the vortex lattice and, paradoxically, induces superconducting state with enhanced critical current. Such magnetized state of the dots (which is metastable in zero field) can exist in zero magnetic field due to large barriers for the dot magnetization reversal. It vanishes only after annealing.

In conclusion, we have shown that the interaction between magnetic dots and superconducting vortices leads to new types of states and transitions between them. We believe that this new kind of interplay between superconductivity and magnetism opens broad prospects for fundamental study and applications.

This work was partly supported by the grants NSF DMR-97-05182 and THECB ARP 010366-003 and by Ministère de l'Enseignement Supérieur et de la Recherche. It is a pleasure to acknowledge discussions with B.Barbara, D.Givord, I.Schuller, D. Naugle and P.Molho. I.L. is thankful to P.Molho for kind hospitality extended to him during his stay at Laboratoire de Magnetisme Louis Neel in Grenoble where part of this work has been done.

* Also at Institute of Physics, 252028 Kiev, Ukraine

[1] O.Geoffroy, D.Givord, Y.Otani, B.Pannetier, and F.Ossart, J. Magn. Magn. Mater. 121, 223, (1993).

[2] W.Wernsdorfer, K.Hasselbach, A. Sulpice, A.Benoit, J.E. Wegrowe, L.Thomas, B.Barbara, and D.Mailly, Phys. Rev. B53, 3341, (1996).

[3] J.I.Martin, M.Velez, J.Nogues, and I.K.Schuller, Phys. Rev. Lett 79, 1929, (1997).

[4] P.C.E.Stamp, E.M.Chudnovsky, and B.Barbara, Int.J.Mod.Phys.B6, 1355, (1992).

[5] Y.Otani, B.Pannetier, J.P.Nozieres, and D.Givord, J. Magn. Magn. Mater. 126, 622, (1993).

[6] Y.Nozaki, Y.Otani, K.Runge, H.Miyajima, B.Pannetier, J.P.Nozieres, and G.Fillon, J. Appl. Phys. 79, 8571, (1996).

[7] R.Allenspach J. Magn. Magn. Mater. 129, 160 (1995).

[8] D.Givord, private communication.

[9] B.Barbara, private communication.

[10] A.A.Abrikosov, Sov.Phys. JETP, 19, 988 (1964) .

[11] A.A.Abrikosov, Introduction to the Theory of Metals, North Holland, Amsterdam,1986.

[12] I.S. Gradstein and I.M. Ryzik Table of integrals, series, and products, 5th ed., Academic Press, Boston 1994.

[13] V.L. Berezinskii, Zh. Eksp. Teor. Fiz., 61, 1144 (1972) (Sov.Phys. JETP, 34, 610 (1972)).

[14] J.M. Kosterlitz and D. Thouless, J. Phys. C6, 1181 (1973); J.M. Kosterlitz, J. Phys. C7, 1046 (1974).

[15] D.Nelson, in Phase Transition and Critical Phenomena (C. Domb and J.L.Lebowitz, eds.). V.7, (1983).

[16] S.Doniach and B.A.Huberman Phys.Rev.Lett., 42, 1169 
(1979).

[17] B.I.Halperin and D.R.Nelson J.Low Temp.Phys., 36, 599 (1979).

[18] P.Minnhagen Rev.Mod.Phys., 59, 1001 (1987).

[19] M. Tinkham, Introduction to Superconductivity McGrawHill, New York, 1975.
[20] D.S. Fisher, D. Friedan, Z.Qiu, S.J. Shenker and S.H. Shenker, Phys. Rev. A 31, 3841 (1985).

[21] V.E. Kravtsov I.V. Lerner and V.I. Yudson, Zh. Eksp. Teor. Fiz. 91, 569 (1986), (Sov.Phys. JETP, 64, 336 (1986)). 